\title{
Combination of acid phosphatase positivity and rimmed vacuoles as useful markers in the diagnosis of adult-onset Pompe disease lacking specific clinical and pathological features
}

\author{
Claire Dolfus ${ }^{1}$, Jean-Philippe Simon ${ }^{2}$, Gérard Landemore ${ }^{1}$, François Leroy ${ }^{3}$, Françoise Chapon ${ }^{2}$ \\ ${ }^{1}$ Department of Pathology, University Hospital of Caen, ${ }^{2}$ Department of Pathology and Neuromuscular Competence Center, \\ University Hospital of Caen, ${ }^{3}$ Neuromuscular Competence Center, University Hospital of Caen, France
}

\begin{abstract}
Introduction: The clinical and histological presentations of the adult form of Pompe disease may be atypical. In such cases, identifying histological signs that point to the diagnosis would be crucial to avoid a delay in care. The aim of our study was to investigate the presence of rimmed vacuoles and acid phosphatase positivity in muscle biopsies of patients with late-onset Pompe disease.

Material and methods: We retrospectively studied muscle biopsies of all cases of the adult form of Pompe disease diagnosed at the University Hospital of Caen. Three of these four cases showed atypical clinical signs, and diagnosis was established tardily based on family history or systematic analysis of acid maltase activity.

Results: All biopsies showed some rimmed vacuoles. The acid phosphatase reaction showed positive inclusions and labelled vacuoles in biopsies of all patients.

Conclusions: The presence of rimmed vacuoles and acid phosphatase positivity in muscle biopsy should suggest the diagnosis of the adult form of Pompe disease, this is decisive since effective therapy is available.
\end{abstract}

Key words: muscle, storage, alpha-glucosidase, Pompe, lysosomal, pathology, rimmed, vacuole, phosphatase.

\section{Introduction}

Pompe disease is an autosomal recessive glycogen (glycogen storage disease type II) that results from a deficiency of alpha-glucosidase (GAA) or acid maltase, a lysosomal enzyme. This enzymatic deficiency generates an abnormal glycogen accumulation mainly in muscle cells. Different phenotypes are reported: infantile, juvenile and adult [3].

The adult form is classically characterized by slowly progressive motor weakness that predom- inantly affects the pelvic limb girdle and by respiratory failure with diaphragmatic paralysis [5]. Since the disease progression can be slowed down by a replacement enzyme therapy (Myozyme ${ }^{\mathrm{TM}}$ ) [14], it is important to get an early diagnosis. The clinical diagnosis is confirmed by an assay of GAA enzyme activity in blood [1]. In some cases, however, the clinical presentation is not obvious, specific or misleading so examination of muscle biopsy is required to raise the suspicion of Pompe disease [13]. 
Histological aspects of Pompe disease in striated muscle are usually cytoplasmic vacuoles, periodic acid Schiff (PAS) positive and highly reactive to acid phosphatase indicating their lysosomal nature [17]. The ultrastructural study confirms the glycogenic nature of the vacuolar content [8]. Other lesions such as segmented fibers, fibers in necrosis/regeneration [15] or acid phosphatase positive inclusions are more rarely observed [18]. An inflammatory lymphocytic infiltrate or a type grouping can mimic chronic myositis or neurogenic damage $[6,15]$. Finally, the muscle biopsy can be normal [13].

Surprisingly, we observed, on a late-onset Pompe disease muscle biopsy, rimmed vacuoles in the cytoplasm of muscle fibers. This histologic appearance is not specific and is conventionally a diagnostic criterion of sporadic (s-IBM) or hereditary (h-IBM) inclusion body myositis, oculopharyngeal muscular dystrophy and few myofibrillar myopathies and distal myopathies [2,9].

The aim of our work has been to evaluate the rimmed vacuoles and acid-phosphatase positivity diagnostic interests as new diagnostic histological markers in late-onset Pompe disease. These histolog ical elements have been sought on muscle biopsies from our patients affected by this insidious disease.

\section{Material and methods \\ Patients}

Patient 1 was diagnosed at the age of 34 years following a respiratory distress episode, initially attributed to pulmonary emphysema with large bubbles for which he was operated. Pulmonary function tests, however, put in evidence a restrictive respiratory secondary to a lordosis, a scoliosis and a diaphragmatic paralysis. In addition, clinical examination revealed severe motor deficit in the limb girdle muscles that has evolved over the last twenty years, and an axial deficit. The 10-meter walking test was achieved in 9 seconds, and the Brooke and Vignos scale was scored at 2. Creatine phosphokinase (CPK) was increased to 2 times normal. The electromyography (EMG) showed myogenic plots. The activity of acid maltase was very low and genetic analysis showed two different mutations in the GAA gene (IVS1-13T>G and c.2182_2183delT). The patient was treated by enzyme replacement therapy Myozyme ${ }^{\mathrm{TM}}$ for 4 years which improved and stabilized his muscular capacity, including breathing.
Patient 2, seen for consultation at the age of 62, showed a typical picture of late-onset Pompe disease. He presented at the age of 38 years for spinal pain secondary to an axial deficit responsible for a hyperlordosis associated with limb girdle muscle weakness, and a face deficit that gradually worsened. At the age of 59, a respiratory failure, secondary to diaphragmatic paralysis, required respiratory support. Creatine phosphokinase was increased to 2 times normal. Functional respiratory tests confirmed diaphragmatic paralysis. The biopsy showed typical lesions of Pompe disease such as numerous vacuoles PAS positive. Unexpectedly, rimmed vacuoles were also found. Biochemical analyses indicated a severe acid maltase deficiency (13\%), and genetic analysis revealed two compound heterozygous pathogenic mutations in the GAA gene (IVS1$13 T>G ; c .1447 G>T)$. The patient died at the age of 65 before the initiation of treatment with Myozyme $^{\mathrm{TM}}$.

Patient 3 is a sister of patient 2, 14 years younger. She was seen for the first time at the age of 45 for a bilateral ptosis associated with proximal muscle weakness of the lower limbs, which started around the age of 40. Further assessment revealed an increase in CPK to 2 times normal, and normal electromyography; inconspicuous inequality of fibers size with some segmentations and rimmed vacuoles were found on muscle biopsy. In view of these findings, a diagnosis of oculopharyngeal muscular dystrophy was made. Unfortunately, PABPN1 gene screening did not find any triplet expansion. She then presented an acromegaly secondary to a pituitary microadenoma, as her mother, and a type 2 diabetes. The evolution was marked by the appearance of ophthalmoplegia, worsening of the limb girdle deficit, and a respiratory failure secondary to a diaphragmatic paralysis. The diagnosis of Pompe disease was then made following that of her brother made a year earlier. Acid maltase activity assessed then was very low (12\%), and genetic analysis showed heterozygote mutations on the GAA gene (IVS1-13T>G; c. $1447 G>T$ ) identical to those observed in her brother. A pathogenic mutation in the $L M N A$ gene (c.1930C $>T$ ) was also found. She died at the age of 56 after 42 administrations of Myozyme ${ }^{\mathrm{TM}}$ over 21 months.

Patient 4, diabetic and hypertensive, was diagnosed at the age of 60 when she was seen for the first time for walking and stair climbing difficulties that began at the age of 38 years and evolved very 
slowly. Physical examination revealed mild proximal weakness of all four limbs and severe axial deficit. Creatine phosphokinase was high, at 4 times normal. On the two muscle biopsies that were performed, only rare rimmed vacuoles were found. The patient did not show any respiratory signs, and respiratory function tests were normal. Acid maltase deficiency was discovered following her inclusion in a research protocol in which patients with an atypical clinical picture were all tested for putative Pompe disease [7]. Diagnosis of Pompe disease was then confirmed by the presence of two mutations in the GAA gene (c.1-45T>G in intron 1 and c.1655T $>C$ in exon 12). Once the patient has begun the treatment with Myozyme ${ }^{\mathrm{TM}}$, a dramatic improvement occurred over the next 18 months, followed by stabilization of the disease.

\section{Frozen sections}

All muscle biopsies were frozen and stored at $-80^{\circ} \mathrm{C}$. Cuts of 8 to 12 microns were formed in the cryostat. The stains and enzymatic reactions carried out were: haematoxylin-eosin, Gomori trichrome, periodic acid-Schiff (PAS), acid phosphatase, ATPase, and oxidative reactions.

\section{Electron microscopy}

The samples were successively fixed by glutaraldehyde, post-fixed with osmium tetroxide, dehydrated, and embedded in resin. Semi-thin and ultrafine sections were then made.

Semi-thin sections were stained with toluidine blue, and also with PAS in patient 2.

Table I. Muscle biopsies of patients affected by the adult form of Pompe disease - histology and electronic microscopy

\begin{tabular}{|c|c|c|c|c|c|}
\hline & $\begin{array}{c}\text { Patient } 1 \\
\text { Left quadriceps }\end{array}$ & $\begin{array}{c}\text { Patient } 2 \\
\text { Left quadriceps }\end{array}$ & $\begin{array}{l}\text { Patient } 3 \\
\text { Left deltoid }\end{array}$ & $\begin{array}{c}\text { Patient } 4 \\
\text { Right deltoid }\end{array}$ & $\begin{array}{c}\text { Patient } 4 \\
\text { Left quadriceps }\end{array}$ \\
\hline \multicolumn{6}{|l|}{ Histology } \\
\hline Inequality of fiber size & ++ & ++ & + & + & ++ \\
\hline Necrosis & - & + & - & - & - \\
\hline Inflammatory infiltrate & - & - & - & - & - \\
\hline Internalized nuclei & + & ++ & + & - & - \\
\hline Segmentation & + & + & - & - & Rare \\
\hline Common vacuoles & + & +++ & + & + & + \\
\hline Rimmed vacuoles & + & ++ & + & + & + \\
\hline Eosinophilic inclusions & - & + & + & - & - \\
\hline PAS on frozen block & - & $\begin{array}{l}\text { On a limited } \\
\text { sector }\end{array}$ & - & - & - \\
\hline PAS on resin block & & $\begin{array}{l}\text { Positivity of all } \\
\text { vacuoles }\end{array}$ & & & \\
\hline $\begin{array}{l}\text { Predominance of one type of } \\
\text { fiber }\end{array}$ & Type I & - & - & - & - \\
\hline Acid phosphatase reaction & $\begin{array}{c}\text { Positive } \\
\text { vacuoles and } \\
\text { inclusions }\end{array}$ & $\begin{array}{c}\text { Positive } \\
\text { vacuoles and } \\
\text { inclusions }\end{array}$ & $\begin{array}{c}\text { Positive } \\
\text { vacuoles and } \\
\text { inclusions }\end{array}$ & $\begin{array}{l}\text { Positive } \\
\text { vacuoles }\end{array}$ & $\begin{array}{l}\text { Positive } \\
\text { vacuoles }\end{array}$ \\
\hline \multicolumn{6}{|l|}{ Electronic microscopy } \\
\hline Free cytoplasmic glycogene & +++ & ++ & + & ++ & ++ \\
\hline Autophagic vacuoles & + & +++ & + & ++ & ++ \\
\hline $\begin{array}{l}\text { Intralysosomial glycogene } \\
\text { accumulation }\end{array}$ & + & ++ & + & + & ++ \\
\hline Anormal mitochondria & - & + & - & - & - \\
\hline $\begin{array}{l}\text { Under sarcolemmal } \\
\text { accumulation of mitochondria }\end{array}$ & + & ++ & + & + & ++ \\
\hline Myofibrilla alteration & + & ++ & + & + & ++ \\
\hline
\end{tabular}




\section{Results}

Histological findings on muscle biopsies are shown in Table I. Particularly relevant is the lack of positive PAS vacuoles for 3 of the 4 patients, and the presence of numerous vacuoles in patient 2 in favour of glycogen excess (Fig. 1A), PAS positivity was observed only on semi-thin sections of this patient. Biopsies of the other patients showed some vacuoles, but they were PAS negative.

Furthermore, rimmed vacuoles were present on all biopsies, obviously predominant in patient 2's biopsy (Table I and Fig. 1B-D). The acid phosphatase reaction showed positive inclusions and labelled vacuoles in biopsies of all patients (Fig. 2A-C).

In electronic microscopy, all biopsies included free cytoplasmic glycogen, glycogen loaded lysosomes and autophagic vacuoles (Fig. 3A-B). The lesions were more marked on patient 2's biopsy. Biopsy of case 1 was characterized by a predominance of glycogen in free form.

\section{Discussion}

Our study shows acid phosphatase positive inclusions and rimmed vacuoles in the biopsy of all four patients.

Acid phosphatase positive inclusions or reduced body-like inclusions, that appear as dense globules to electrons in electronic microscopy, are described in the adult form of Pompe disease [16,18]. According to Feeney et al., these structures correspond to lipofuscin inclusions [4]. The present study confirms that the acid phosphatase positive inclusions can be a useful diagnostic marker for Pompe disease in cases without histological evidence, as previously suggested by Tsuburaya et al. [18]. Rimmed vacuoles were present in biopsies of all our patients with late-onset Pompe disease. The rimmed vacuoles are small vacuoles highlighted by basophil grains on hematoxylin-eosin and red grains on Gomori trichrome [12]. According to Nishino et al. [10,12],
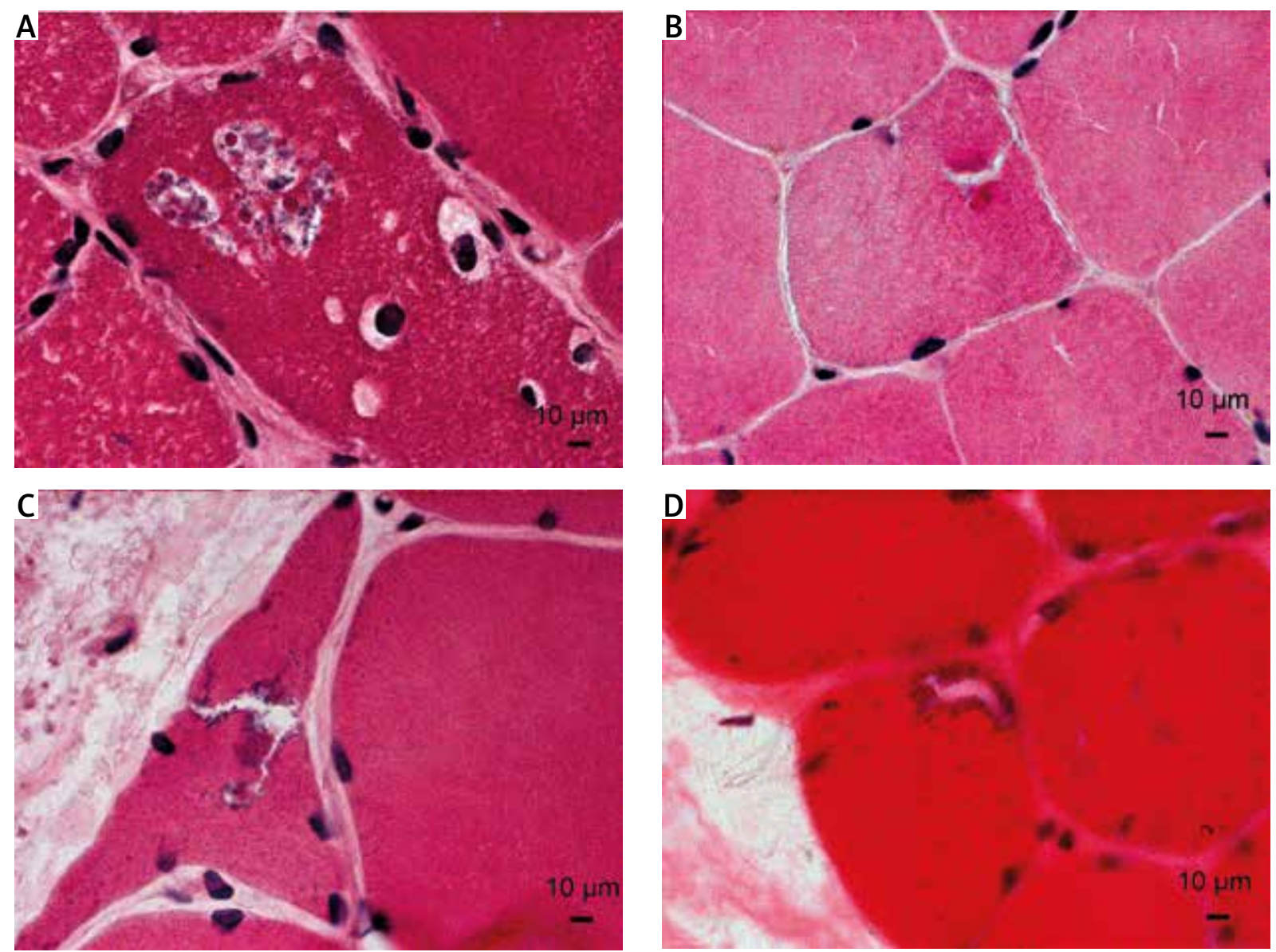

Fig. 1. Hematoxylin-eosin: A) Common vacuoles and vacuoles with a basophilic material on hematoxylin and eosin (patient 2), B-D) Rimmed vacuoles (patient 4, 1 and 3). 

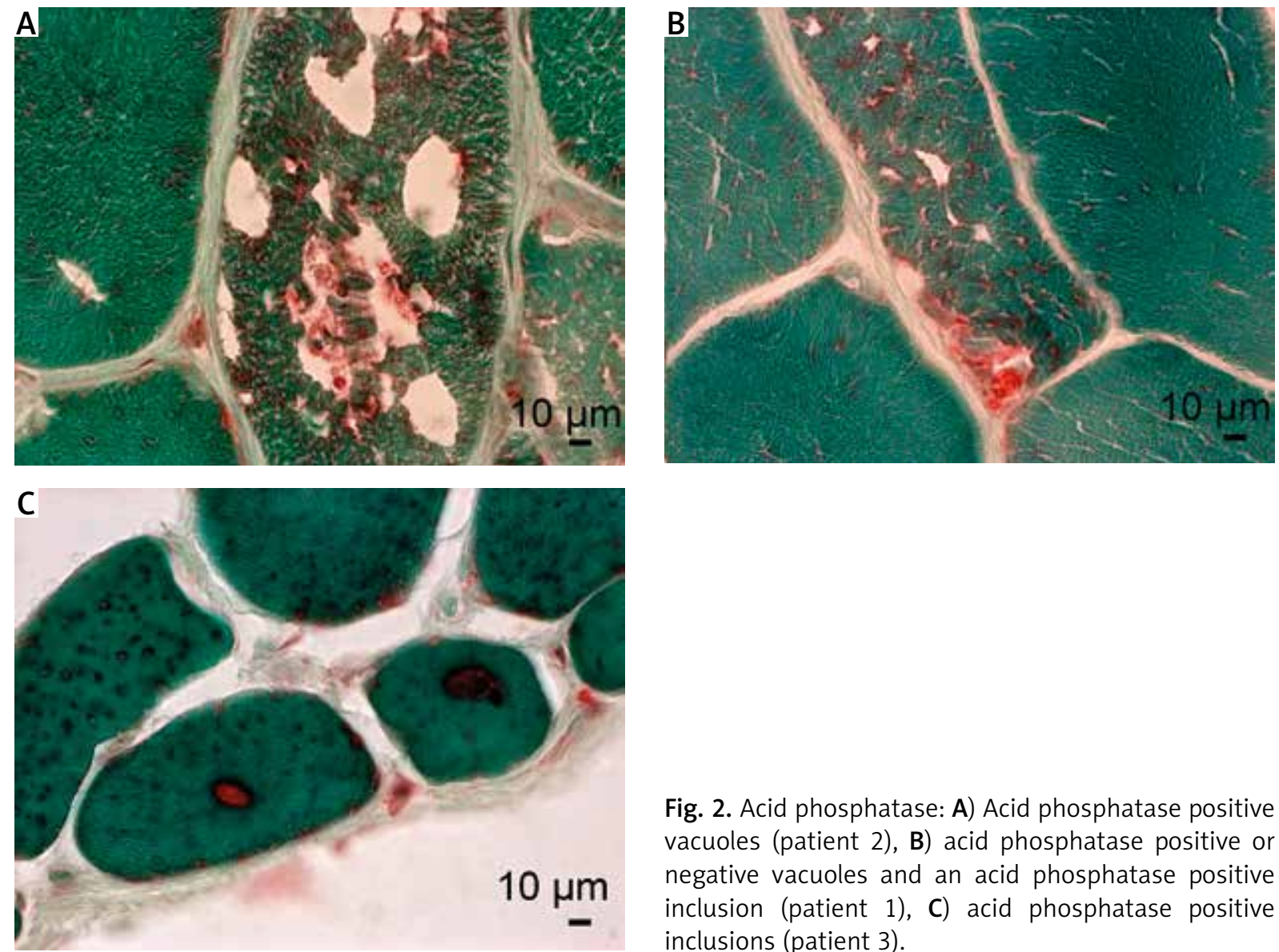

Fig. 2. Acid phosphatase: A) Acid phosphatase positive vacuoles (patient 2), B) acid phosphatase positive or negative vacuoles and an acid phosphatase positive inclusion (patient 1), C) acid phosphatase positive inclusions (patient 3).
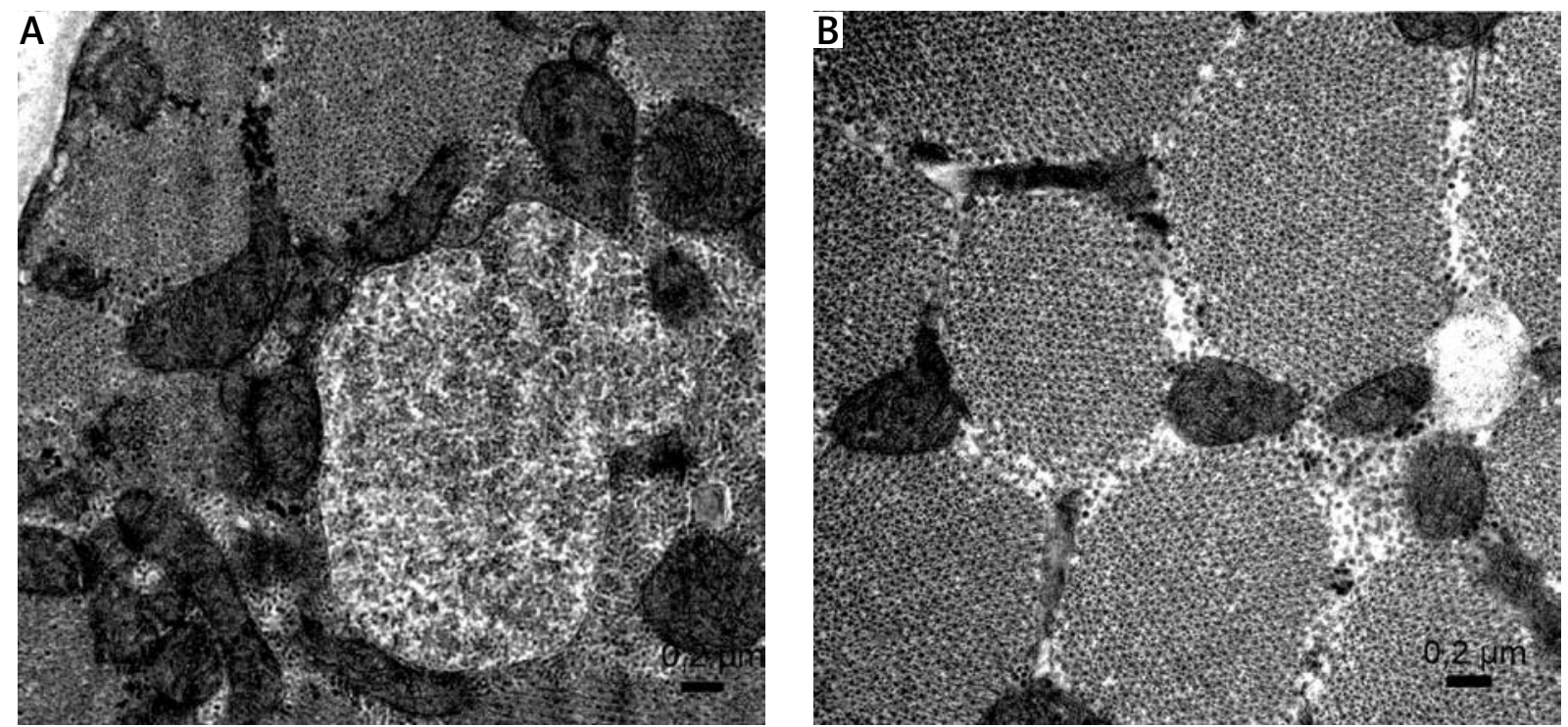

Fig. 3. Electronic microscopy: A) lysosomal accumulation of glycogen (patient 2), B) extra-lysosomal accumulation of glycogen on electronic microscopy (patient 4). 
rimmed vacuoles would be artifacts formed during the coloring process: the rimmed vacuoles would correspond to clusters of autophagic vacuoles coming off the glass slide and regrouped in myofibrils nearby.

Although Nishino reported that some histological aspects observed in Pompe disease look like rimmed vacuoles [11], he does not include this disease in the list of numerous inherited diseases characterized by this histological abnormality (hereditary inclusion body myopathy, distal myopathy with rimmed vacuoles, limb-girdle muscular dystrophy $2 \mathrm{G}$, inclusion body myopathy 3 , limb-girdle muscular dystrophy $1 \mathrm{~A}$, oculopharyngeal muscular dystrophy, desmin myopathy, desmin-related myopathy, tibial muscular dystrophy) [12].

To our knowledge, Schoser et al. [15] are the only ones to have previously reported aspects of "rimmed vacuoles" in muscle biopsies of cases of the adult form of Pompe disease; this was observed in rare biopsies in their cohort of 38 patients.

In our study, biopsies of all patients with late-onset Pompe disease had genuine rimmed vacuoles even when glycogen deposits were moderate or minimal in electronic microscopy (patients $1,2,4$ ).

Based on clinicopathological correlations (ptosis and rimmed vacuoles), our case 2 was initially misdiagnosed with oculopharyngeal dystrophy. Our study highlights that it is thus important for a pathologist to include the Pompe disease in the list of diseases characterized by rimmed vacuoles, especially since effective treatment is available for this disease of acid maltase deficiency.

\section{Conclusions}

The presence of both acid phosphatase positive inclusions and rimmed vacuoles in muscle biopsies should thus suggest a diagnosis of Pompe disease in case of clinically atypical forms, and prompt the clinician to make measurement of enzyme activity and analysis in molecular biology to confirm the diagnosis. These new diagnostic screening markers of Pompe disease should, however, be validated on a larger number of cases.

\section{Acknowledgements}

The authors are grateful to Chantal Chavoix, Caen University Hospital, for her help in editing the manuscript.

\section{Disclosure}

Authors report no conflict of interest.

\section{References}

1. American Association of Neuromuscular \& Electrodiagnostic Medicine. Diagnostic criteria for late-onset (childhood and adult) Pompe disease. Muscle Nerve 2009; 40: 149-160.

2. Dimachkie MM, Barohn RJ. Inclusion body myositis. Curr Neurol Neurosci Rep 2013; 13: 321.

3. DiMauro S, Spiegel R. Progress and problems in muscle glycogenoses. Acta Myol 2011; 30: 96-102.

4. Feeney EJ, Austin S, Chien Y-H, Mandel H, Schoser B, Prater S, Hwu WL, Ralston E, Kishnani PS1, Raben N. The value of muscle biopsies in Pompe disease: identifying lipofuscin inclusions in juvenile- and adult-onset patients. Acta Neuropathol Commun 2014; 2: 2 .

5. Hagemans MLC, Winkel LPF, Van Doorn PA, Hop WJ, Loonen MC, Reuser AJ, Van der Ploeg AT. Clinical manifestation and natural course of late-onset Pompe's disease in 54 Dutch patients. Brain J Neurol 2005; 128 (Pt 3): 671-677.

6. Hobson-Webb LD, Proia AD, Thurberg BL, Banugaria S, Prater SN, Kishnani PS. Autopsy findings in late-onset Pompe disease: a case report and systematic review of the literature. Mol Genet Metab 2012; 106: 462-469.

7. Laforêt P, Laloui K, Granger B, Hamroun D, Taouagh N, Hogrel JY, Orlikowski D, Bouhour F, Lacour A, Salort-Campana E, Penisson-Besnier I, Sacconi S, Zagnoli F, Chapon F, Eymard B, Desnuelle C, Pouget J. The French Pompe registry. Baseline characteristics of a cohort of 126 patients with adult Pompe disease. Rev Neurol (Paris) 2013; 169: 595-602.

8. Lewandowska E, Wierzba-Bobrowicz T, Rola R, Modzelewska J, Stepień T, Lugowska A, Pasennik E, Ryglewicz D. Pathology of skeletal muscle cells in adult-onset glycogenosis type II (Pompe disease): ultrastructural study. Folia Neuropathol 2008; 46: 123-133.

9. Lu H, Luan X, Yuan Y, Dong M, Dong M, Sun W, Yan C. The clinical and myopathological features of oculopharyngodistal myopathy in a Chinese family. Neuropathology 2008; 28: 599-603.

10. Malicdan MCV, Noguchi S, Nishino I. Autophagy in a mouse model of distal myopathy with rimmed vacuoles or hereditary inclusion body myopathy. Autophagy 2007; 3: 396-398.

11. Malicdan MCV, Nishino I. Autophagy in lysosomal myopathies. Brain Pathol 2012; 22: 82-88.

12. Nishino I. Autophagic vacuolar myopathies. Curr Neurol Neurosci Rep 2003; 3: 64-69.

13. Saux A, Laforet P, Pagès AM, Figarella-Branger D, Pellissier JF, Pagès $M$, Labauge $P$. A retrospective study of six patients with late-onset Pompe disease. Rev Neurol (Paris) 2008; 164: 336-342.

14. Schneider I, Hanisch F, Müller T, Schmidt B, Zierz S. Respiratory function in late-onset Pompe disease patients receiving longterm enzyme replacement therapy for more than 48 months. Wien Med Wochenschr 2013; 163: 40-44.

15. Schoser BGH, Müller-Höcker J, Horvath R, Gempel K, Pongratz D, Lochmüller H, Müller-Felber W. Adult-onset glycogen stor- 
age disease type 2: clinico-pathological phenotype revisited.

Neuropathol Appl Neurobiol 2007; 33: 544-559.

16. Sharma MC, Schultze C, von Moers A, Stoltenburg-Didinger G, Shin YS, Podskarbi T, Isenhardt K, Tews DS, Goebel HH. Delayed or late-onset type II glycogenosis with globular inclusions. Acta Neuropathol 2005; 110: 151-157.

17. Steps forward in Pompe Disease. Abstracts of the 4th European Symposium. November 19-20, 2010. London, United Kingdom. Clin Ther 2011; 33 (6 Suppl 1): S1-40.

18. Tsuburaya RS, Monma K, Oya Y, Nakayama T, Fukuda T, Sugie H, Hayashi YK, Nonaka I, Nishino I. Acid phosphatasepositive globular inclusions is a good diagnostic marker for two patients with adult-onset Pompe disease lacking disease specific pathology. Neuromuscul Disord 2012; 22: 389-393. 
